\title{
Synthesis and Characterization of Quasi-Solid-State Organic-Inorganic Hybrid Electrolytes for Electrochemical Gas Sensors
}

\author{
Sang-Hyung Kim ${ }^{1}$, Seung Hark Park', Dong-Kwan Han ${ }^{1}$, Dong-Won Kim ${ }^{1}$ \\ Dong-Yun Lee ${ }^{2}$, Sang-Do Han', Jin-Seong Park ${ }^{3}$ \\ ${ }^{1}$ Department of Chemical Engineering, Hanyang University, Seoul 04763, Korea \\ ${ }^{2}$ ShinWoo Electronics Co.Ltd, Hwa Seoung 445-915, Korea \\ ${ }^{3}$ Deartment of Materials Engineering, Chosun University, Kwangju 501-759, Korea \\ E-mail: dongwonkim@hanyang.ac.kr
}

\begin{abstract}
The main goal of this work is to verify if use of quasi-solid-state electrolyte can improve the performance of the electrochemical gas sensor for detecting the toxic gases. In order to achieve this goal, the quasi-solid-state organic-inorganic hybrid electrolytes with high ionic conductivity are synthesized by using reactive silica particles, tetra(ethylene glycol) diacrylate and sulfuric acid, and their electrochemical properties are investigated. The quasi solid-state electrolytes are then applied to electrochemical gas sensors, and the sensor responses are measured. The results obtained in the gas sensors with quasi solid-state electrolyte are compared with response obtained in conventional electrochemical gas sensors employing liquid electrolyte.
\end{abstract}

Key words: electrochemical gas sensor, quasi-solid-state electrolyte, hybrid electrolyte, sensor response, toxic gas

Electrochemical gas sensor is a device that measures the concentration of a target gas by oxidizing or reducing the gas at an electrode and measuring the resulting current. They have fast response, good sensitivity and selectivity, and thus are suitable for sensing toxic gases. These sensors usually consist of two or three electrodes in contact with an electrolyte. The working electrode contacts both the electrolyte and the ambient air. The electrolytes commonly used in the electrochemical gas sensors are an acid-based and alkaline-based electrolyte. Since these liquid electrolytes are directly contacted with air, their characteristics can be affected by the external environment such as humidity and temperature. Moreover, the reliability and stability of electrochemical gas sensor employing liquid electrolyte is not good due to the leakage of liquid electrolyte. In order to solve these issues, different kinds of solid electrolytes such as inorganic and polymer have been actively studied as an electrolyte for electrochemical gas sensor operable at low temperatures [1,2].

In this study, we prepared highly conductive quasi-solid-state organic-inorganic hybrid electrolytes by chemical cross-linking of the solution containing silica particles, tetra(ethylene glycol) diacrylate as the crosslinking agents and sulfuric acid as a supporting electrolyte, and their electrochemical properties were investigated. The electrochemical gas sensor was then fabricated by employing a quasi-solid-state hybrid electrolyte without solvent leakage and Pt-based electrode. Their gas sensing properties were investigated and compared with responses obtained in conventional electrochemical gas sensors employing liquid electrolyte.

\section{References}

[1] N. Miura, N. Yamazoe, Development of New Chemical Sensors Based on Low-temperature Proton Conductors, Solid State Ionics 53-56, 975-982 (1992); doi: 10.1016/0167-2738.

[2] T. Hyodo, C. Ishibashi, K. Matsuo, K. Kaneyasu, $\mathrm{H}$. Yanagi, Y. Shimizu, $\mathrm{CO}$ and $\mathrm{CO}_{2}$ Sensing Properties of Electrochemical Gas Sensors using an Anion-conducting Polymer as an Electrolyte, Electrochim. Acta 82, 19-25 (2012); doi:10.1016/j.electacta.2012.03.142. 